\title{
Odd-Even Staggering of Nuclear Masses: Pairing or Shape Effect?
}

\author{
W. Satuła, ${ }^{1-3}$ J. Dobaczewski, ${ }^{1-3}$ and W. Nazarewicz ${ }^{2-4}$ \\ ${ }^{1}$ Joint Institute for Heavy Ion Research, Oak Ridge, Tennessee 37831 \\ ${ }^{2}$ Department of Physics, University of Tennessee, Knoxville, Tennessee 37996 \\ ${ }^{3}$ Institute of Theoretical Physics, University of Warsaw, ul. Hoża 69, PL-00-681 Warsaw, Poland \\ ${ }^{4}$ Physics Division, Oak Ridge National Laboratory, Oak Ridge, Tennessee 37831
}

(September 4, 2018)

\begin{abstract}
The odd-even staggering of nuclear masses was recognized in the early days of nuclear physics. Recently, a similar effect was discovered in other finite fermion systems, such as ultrasmall metallic grains and metal clusters. It is believed that the staggering in nuclei and grains is primarily due to pairing correlations (superconductivity), while in clusters it is caused by the Jahn-Teller effect. We find that, for light and medium-mass nuclei, the staggering has two components. The first one originates from pairing while the second, comparable in magnitude, has its roots in the deformed mean field.

PACS number(s): 21.10.Dr, 21.10.Pc, 21.60.Jz, 71.15.Mb
\end{abstract}

The odd-even staggering (OES) of binding energies has been observed in several finite many-fermion systems such as nuclei [1], ultrasmall superconducting grains [2], and metal clusters [3]. It manifests itself in the fact that the binding energy of a system with an odd particle number is lower than the arithmetic mean of the energies of the two neighboring even-particle-number systems.

In atomic nuclei, the OES is usually attributed to the existence of nucleonic pairing correlations [4.57. A similar scenario has been proposed for metallic grains (see Refs. [6,7] and references quoted therein). In both cases, the finite-size effects are important, and the Cooper pairing is well described in terms of the parity-number-conserving quasiparticle approach. Although the motion of electrons in metals is very different from that of nucleons in nuclei, the mechanism behind electronic and nucleonic superconductivity (presence of attractive residual interaction which gives rise to a correlated many-fermion system) is indeed very similar [8,9].

So far, no evidence has been found for superconductivity in alkali metal clusters [10], and the OES of binding energies in such systems is attributed to a very different mechanism. Namely, it is believed to have its origin in the Jahn-Teller effect which, by breaking the spherical symmetry of the mean field, gives rise to deformed singleparticle orbitals [11,12]. Recently, Häkkinen et al. [13], using the density-functional theory, argued that light alkali-metal clusters and light $N=Z$ nuclei have a similar pattern of OES, irrespective of differences in the interactions between the fermions. Hence, they concluded that the OES in small nuclei appears to be a mere deformation effect rather than a consequence of pairing.

The main objective of this study is to analyze the phenomenon of OES in nuclei from the microscopic perspective. Guided by self-consistent calculations, we make an attempt to determine and separate the pairing and meanfield contributions to the experimental OES.

In the independent quasiparticle (BCS) picture [14, the gap parameter, $\Delta$, can be related to the binding energies of three adjacent systems. Assuming that the binding energies of even systems and those of odd systems are locally smooth functions of the particle number $N$, the quantity

$$
\Delta^{(3)}(N) \equiv \frac{\pi_{N}}{2}[B(N-1)+B(N+1)-2 B(N)]
$$

is often interpreted as a measure of the empirical pairing gap. In Eq. (1),$\pi_{N}=(-1)^{N}$ is the number parity and $B(N)$ is the (negative) binding energy of a system with $N$ particles. Indeed, assuming that the ground state of the odd- $N$ system is a pure one-quasiparticle state, one has $\Delta^{(3)}(N) \approx E_{k} \approx \Delta$, where $E_{k}=\sqrt{\left(e_{k}-\lambda\right)^{2}+\Delta^{2}}$ is the lowest BCS quasiparticle energy, $e_{k}$ is the energy of the single-particle orbital occupied by the odd nucleon, and $\lambda(=d B / d N)$ is the Fermi energy.

Another commonly used binding-energy relation for $\Delta$ is the four-point expression [15],

$$
\begin{aligned}
\Delta^{(4)}(N) & \equiv \frac{\pi_{N}}{4}[3 B(N-1)-3 B(N)-B(N-2) \\
& +B(N+1)]=\frac{1}{2}\left[\Delta^{(3)}(N)+\Delta^{(3)}(N-1)\right],
\end{aligned}
$$

which averages the $\Delta^{(3)}$ values in adjacent even and odd systems. In nuclei, because there are two kinds of particles, neutrons and protons, $\Delta$ is calculated along the isotopic or isotonic chains [5]. The resulting pairing gaps are denoted as $\Delta_{\nu}$ and $\Delta_{\pi}$, respectively. In what follows we argue that different physical effects determine the behavior of $\Delta^{(3)}(N)$ for odd and even particle numbers. Hence, the interpretation of the average (2) in terms of the pairing gap can be misleading.

To investigate the Jahn-Teller component of the OES, we performed the Hartree-Fock (HF) calculations without pairing for nuclei with $Z=9 \div 28$ and $N-Z=-2 \div 6$. The HFODD code (v1.75) [16] and two different Skyrme parametrizations, SIII [17] and SLy4 [18, were employed. 
For each Skyrme force, two sets of calculations were carried out: either requiring time-reversal symmetry to be conserved or not. (The time-odd components appear in the mean fields of odd and odd-odd systems.) Since, according to our calculations, the time-odd terms do not affect qualitatively the OES, the results presented in this work were obtained by neglecting these terms.

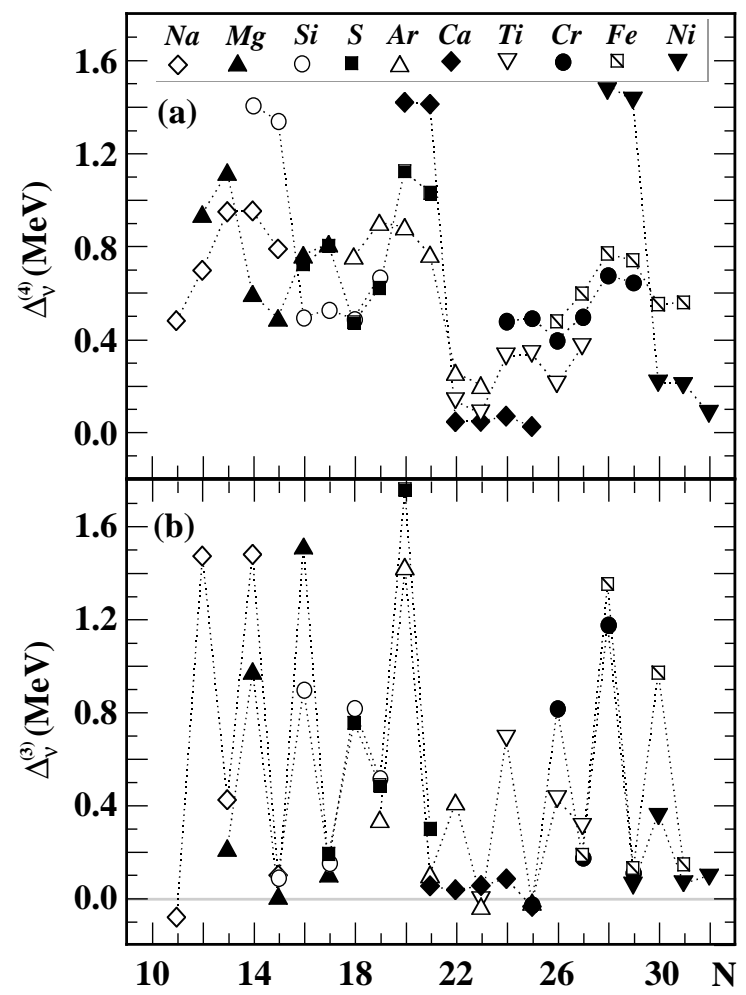

FIG. 1. $\Delta_{\nu}^{(4)}$ (a) and $\Delta_{\nu}^{(3)}$ (b) calculated in the SIII HF model without pairing as a function of neutron number.

Figure 1 a displays the theoretical values of $\Delta_{\nu}^{(4)}$ obtained from binding energies in the SIII model. The results obtained with the SLy4 parametrization are strikingly similar, in spite of the fact that the total binding energies predicted by these two forces show large differences, approaching $4 \mathrm{MeV}$ in some cases. This result suggests that, unlike the total binding energy, $\Delta^{(4)}$ weakly depends on the effective interaction.

Results shown in Fig. 11a demonstrate that the selfconsistent mean-field theory without pairing does indeed predict the OES according to the criterion (2). The effect is sizable: theoretical values of $\Delta_{\nu}^{(4)}$ reach $30 \%$ to $50 \%$ of the empirical OES and they, on average, gradually decrease with mass. A rather complicated pattern of $\Delta_{\nu}^{(4)}$ can be easily explained by looking at values of $\Delta_{\nu}^{(3)}$ presented in Fig. 1 1 b. Values of $\Delta_{\nu}^{(3)}$ are large for $\pi_{N}=+1$ and very small for $\pi_{N}=-1$; hence the averages $\Delta_{\nu}^{(4)}$ (2) simply reflect the simple pattern of $\Delta_{\nu}^{(3)}$. (The behavior of $\Delta_{\pi}^{(3)}$ shows a very similar pattern. Also much the same results were obtained with the SLy4 force.)

We are now in a position to trace the pattern of $\Delta_{\nu}^{(3)}$, shown in Fig. 1 b, back to properties of the deformed mean field. Indeed, Eq. (11) represents the second-order difference with respect to $N$, i.e.,

$$
2 \pi_{N} \Delta^{(3)}(N) \approx \frac{\partial^{2} B}{\partial N^{2}}=\frac{\partial \lambda}{\partial N}=\frac{1}{g(\lambda)},
$$

where $g(e) \equiv d N / d e$ is the single-particle level density. Consequently, in the absence of OES due to pairing, $\Delta^{(3)}$ represents the variation of the Fermi energy with particle number. In the case of a degenerate shell, the Fermi energy does not change with $N$ ( $\lambda$ lies on the last occupied level) and $\Delta^{(3)}=0$. The change in $\lambda$ takes place when the valence shell $e_{n}(N=2 n)$ is completely filled and the higher shell, $e_{n+1}$, needs to be occupied. In this case, corresponding to $\pi_{N}=+1, d \lambda / d N \approx e_{n+1}-e_{n}$, and $\Delta^{(3)} \approx\left(e_{n}-\right.$ $\left.e_{n+1}\right) / 2$. That is, in the absence of pairing correlations, $\Delta^{(3)}$ becomes a measure of a gap in the single-particle spectrum. This single-particle mechanism behind the OES was early noticed in Ref. [5] and subsequently employed in Refs. 11, 12, to explain the OES in metal clusters. The alternating behavior of $\Delta^{(3)}$ in Fig. 1 $1 \mathrm{~b}$ comes from the twofold Kramers degeneracy of the deformed single-particle energy levels. Indeed, the spherical symmetry of the mean-field potential, which gives rise to a $(2 j+1)$-fold degeneracy of single-particle levels, is preserved only for doubly magic nuclei. For open-shell systems, spherical symmetry is spontaneously broken by the Jahn-Teller mechanism, and the ground state is characterized by the deformed mean field, cf. Refs. [19,20].

Results of the self-consistent calculations for nuclei and metal clusters can be understood in terms of the macroscopic-microscopic shell-correction method. In this approach, which can be viewed as an approximation to the HF treatment 21], the total binding energy can be written as $B=E_{\mathrm{sp}}-\tilde{E}_{\mathrm{sp}}+E_{\text {macro }}$, where

$$
E_{\mathrm{sp}}=\sum_{k=1}^{A} e_{k}
$$

is the shell-model energy (sum of single-particle energies of occupied states), $\tilde{E}_{\mathrm{sp}}$ is the Strutinsky-averaged shellmodel energy, and $E_{\text {macro }}$ stands for the macroscopic liquid-drop energy.

The liquid-drop contributions to the second difference (3) differ for nuclei and clusters. For nuclei, the main effect comes from the symmetry energy term 22]. Assuming the symmetry energy coefficient $a_{I}=38 \mathrm{MeV}$ (the value appropriate for light nuclei 23|) the nuclear liquiddrop curvature contribution to (3) is $38 / A \mathrm{MeV}$, while the contribution of the surface-energy term is much smaller. On the contrary, in alkali-metal clusters, the leading contribution from the liquid-drop term to (3) comes from the surface energy and is negligible. Indeed, taking the typical value of the surface-tension coefficient for the bulk 
$\mathrm{Na}, \sigma \approx 0.01 \mathrm{eV} / \AA^{2}$, one obtains a very small correction $\partial^{2} E_{\text {macro }} / \partial N^{2} \approx-0.15 / N^{4 / 3} \mathrm{eV}$.

In order to evaluate the curvature contribution from $\tilde{E}_{\mathrm{sp}}$, one needs to estimate the average single-particle level density at the Fermi energy, $g(\lambda)$ [see Eq. (3)]. In the nuclear case $g(\lambda)=3 a / \pi^{2}$, where $a$ is the level density parameter [5]. Experimentally, $a \approx A / 8 \mathrm{MeV}$ for light nuclei, and this agrees well with the estimate based on realistic potentials 24. Since in our HF calculations the effective mass is less than one, the level density parameter should be additionally multiplied by the effectivemass factor: $m^{*} / m=0.76$ and 0.7 for SIII and SLy4, respectively. Consequently, the corresponding curvature contribution becomes $-1 / g(\lambda) \approx-36 / A \mathrm{MeV}$, and nearly cancels out the liquid-drop contribution. One can, therefore, conclude that the leading contribution to the HF values of $\Delta^{(3)}$ shown in Fig. 1 $1 \mathrm{~b}$ comes from the singleparticle sum (荬),

$$
\delta e \equiv \Delta_{\mathrm{sp}}^{(3)}(N) \approx \frac{1}{4}\left(1+\pi_{N}\right)\left(e_{n+1}-e_{n}\right),
$$

provided that one neglects small shifts in the singleparticle energies due to variations of the mean field (e.g., deformation changes) when increasing the particle number from $N$ to $N+1$. (The effect of the mean-field variations is seen in Fig. 1 $\mathrm{b}$ for particle numbers 13, 19, and 27 , i.e., around magic gaps where the shape transitions take place.) Quantity (5) represents, therefore, the effective single-particle spacing between nucleonic energy levels.

The above conclusion is not true for alkali-metal clusters. The level density for alkali-metal clusters can be estimated following Ref. [5]: $g(\lambda)=0.154 m_{e} r_{0}^{2} N / \hbar^{2}$. By taking $r_{0}=2.17 \AA$ (the value corresponding to the density of bulk $\mathrm{Na}$ at $500^{\circ} \mathrm{K}$ ), one obtains $1 / g(\lambda) \approx 1.4 / \mathrm{NeV}$. (This result is fairly close to the Fermi-gas estimate of $1 / g(\lambda) \approx 2.15 / N \mathrm{eV}$, assuming $\lambda=3.23 \mathrm{eV}$.) Contrary to the nuclear case, since the liquid-drop component of the second difference is very small, the smoothed-energy term strongly contributes to $\Delta^{(3)}$. Moreover, the typical single-particle splitting due to cluster deformation is of the order of $0.2 \mathrm{eV}$ [25], i.e., it is very similar to the value of $1 / g(\lambda)$. For example, in Fig. 4 of Ref. [13], the calculated OES parameter $2 \pi_{N} \Delta^{(3)}$ oscillates around zero - in contrast to the nuclear case presented in Fig. 1 $1 \mathrm{~b}$. This is because the smoothed-energy contribution to the OES, $-1 / g(\lambda)$, introduces a negative shift of the same order as term (5).

Since in nuclei the mean-field contribution $\delta e$ is very small for $\pi_{N}=-1$, both pairing and mean-field components of OES can be extracted from binding energies by using the three-point filter $\Delta^{(3)}$. As illustrated schematically in Fig. 2, values of $\Delta^{(3)}$ calculated at odd values of $N$ can be associated with the pairing effect [26],

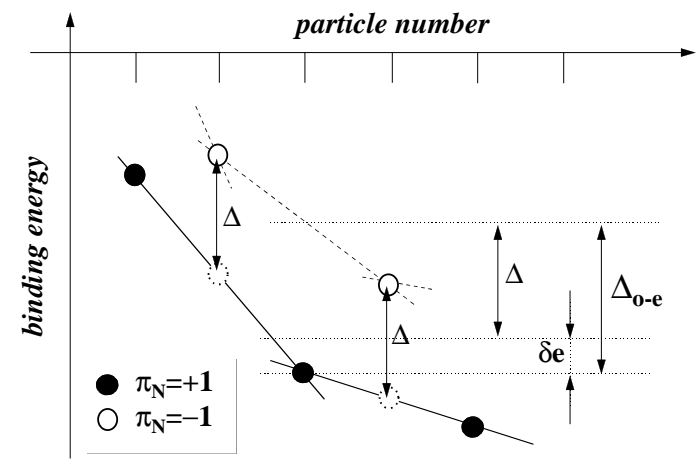

FIG. 2. Schematic illustration of various contributions to the OES. The odd-even energy difference, $\Delta_{\mathrm{o}-\mathrm{e}}$, is decomposed into the pairing part, $\Delta$, and the mean-field part, $\delta e$.

$$
\Delta_{\nu}(N) \equiv \Delta_{\nu}^{(3)}(N=2 n+1),
$$

while the differences of $\Delta^{(3)}$ at adjacent even and odd values of $N$ give information about the single-particle spectra,

$$
e_{n+1}-e_{n}=2\left[\Delta_{\nu}^{(3)}(N=2 n)-\Delta_{\nu}^{(3)}(N=2 n+1)\right] .
$$

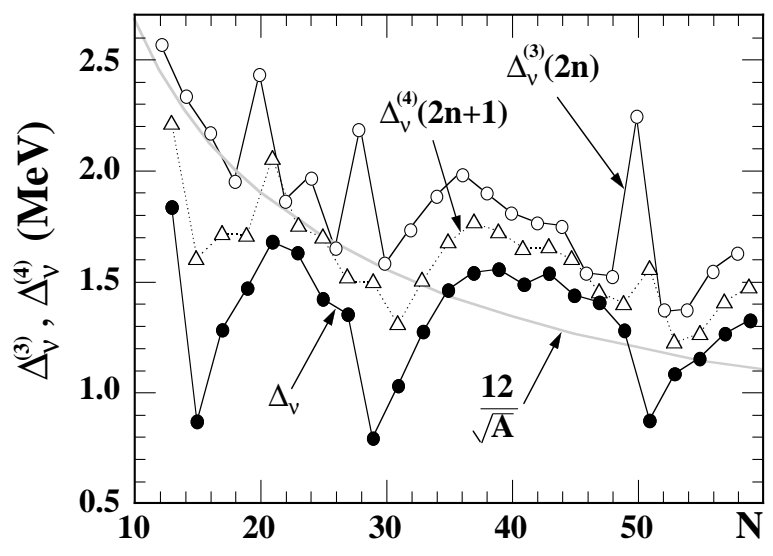

FIG. 3. Experimental values of $\Delta_{\nu}(N)=\Delta_{\nu}^{(3)}(N)$ for $N$-odd (filled circles), $\Delta_{\nu}^{(3)}$ for $N$-even (open circles), and $\Delta_{\nu}^{(4)}$ for $N$-odd (open triangles). The thick gray line indicates the average trend, $\tilde{\Delta}=12 / \sqrt{A}$. Each point represents the arithmetic mean over several even- $Z$ isotones.

The neutron pairing gaps (5) extracted from the experimental binding energies are shown in Fig. 3. The expected quenching of neutron pairing at magic (or semimagic) particle numbers $N=14,28$, and 50 is clearly seen. (Interestingly, the minimum at $N=20$ is absent.)

The experimental values of $\Delta_{\nu}^{(3)}$ at even neutron numbers are systematically shifted with respect to $\Delta_{\nu}$. Since the differences (7) reflect the mean-field contributions to the OES, they sharply peak at magic numbers. In the 
experimental values of $\Delta_{\nu}^{(4)}$ the magic gaps are almost invisible. This is so because the effects of a large singleparticle gap and quenched pairing cancel out in the averages (2). Since the commonly used smooth dependence of average pairing gap on mass number, $\tilde{\Delta}=12 / \sqrt{A}$, was fitted to $\Delta^{(4)}$, and not to $\Delta[27]$, the values of $\tilde{\Delta}$ are overestimated, especially for light systems. As seen in Fig. 3, the values of $\Delta_{\nu}$ in the middle of the $s d$ and $p f$ shells are fairly similar, and are not consistent with the $A^{-1 / 2}$ trend.

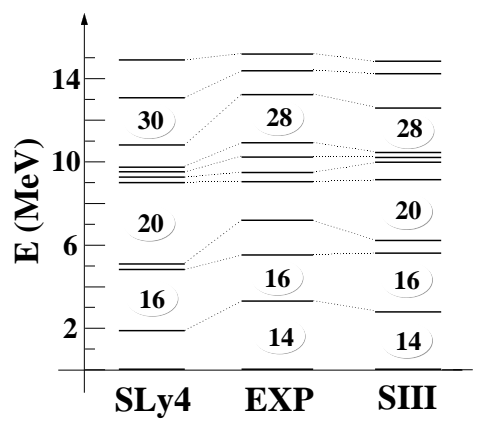

FIG. 4. Experimental and calculated effective single-particle spectrum extracted by means of relation (7) for $N-Z=4$.

Finally, we have extracted from the data the meanfield contributions to the OES according to Eq. (7). As an example, in Fig. 目 they are compared with the HF results for the $N-Z=4$ chains. In spite of the fact that no pairing correlations have been considered in calculations, a good agreement between the experimental and theoretical levels is obtained. The fact that theoretical and experimental energy scales agree is a consequence of the fact that both SIII and SLy4 reproduce fairly well the symmetry energy. The level bunching predicted between the $N=20$ and $N=28$ gaps reflects small calculated equilibrium deformations in these $1 f_{7 / 2}$-shell nuclei, which nicely agrees with a similar grouping of levels seen in experiment.

In summary, our analysis does not confirm the recent suggestion [13] that the OES in light nuclei is a mere reflection of the deformed mean field. We have demonstrated that the OES in light atomic nuclei is strongly affected by both nucleonic pairing and the deformed meanfield. A method has been proposed to extract the pairing contribution to the OES from experimental data. The experimental pairing gaps show a weaker $A$-dependence than that obtained previously. Since the fourth-order mass difference $\Delta^{(4)}$ is strongly affected by the meanfield contribution, it is not a good measure of pairing correlations, at least in light nuclei.

In our discussion we have not discussed the singular behavior of binding energies of the $N=Z$ nuclei, known as the Wigner energy, which dramatically influences the binding-energy relations (11) and (2) 228,29,23]. Consequently, the OES near the $N=Z$ line has an additional third component originating from the neutron-proton correlations. In particular, the OES parameter discussed in Ref. 13], which is based on binding energy differences of even-even and odd-odd $N=Z$ nuclei (hence it does not represent any pairing gap), is strongly perturbed by this third component [29].

Useful discussions with S. Frauendorf are gratefully acknowledged. This research was supported in part by the U.S. Department of Energy under Contract Nos. DEFG02-96ER40963 (University of Tennessee), DE-FG0587ER40361 (Joint Institute for Heavy Ion Research), DE-AC05-96OR22464 with Lockheed Martin Energy Research Corp. (Oak Ridge National Laboratory), and by the Polish Committee for Scientific Research (KBN) under Contract No. 2 P03B 04014.

[1] W. Heisenberg, Z. Phys. 78, 156 (1932).

[2] C.T. Black et al., Phys. Rev. Lett. 76, 688 (1996).

[3] W.A. de Heer, Rev. Mod. Phys. 65, 611 (1993).

[4] A. Bohr et al., Phys. Rev. 110, 936 (1958).

[5] A. Bohr and B.R. Mottelson, Nuclear Structure, vol. 1 (W.A. Benjamin, New York, 1969).

[6] R. Rossignoli et al., Phys. Rev. Lett. 80, 1853 (1998).

[7] R. Balian et al., cond-mat/9802006.

[8] A. Bohr and B.R. Mottelson, Nuclear Structure, vol. 2 (W.A. Benjamin, New York, 1975).

[9] H.J. Lipkin, APS News, January 1998.

[10] N.K. Kuzmenko et al., Nuovo Cimento 18D, 645 (1996).

[11] K. Clemenger, Phys. Rev. B32, 1359 (1985).

[12] M. Manninen et al., Z. Phys. D31, 259 (1994).

[13] H. Häkkinen et al., Phys. Rev. Lett. 78, 1034 (1997).

[14] J. Bardeen et al., Phys. Rev. 108, 1175 (1957).

[15] S.G. Nilsson and O. Prior, Mat. Fys. Medd. Dan. Vid. Selsk. 32, No. 16 (1961).

[16] J. Dobaczewski and J. Dudek, Comp. Phys. Commun. 102, 166, 183 (1997), and to be published.

[17] M. Beiner et al., Nucl. Phys. A238, 29 (1975).

[18] E. Chabanat et al., Nucl. Phys. A627, 710 (1997).

[19] P.-G. Reinhard and E.W. Otten, Nucl. Phys. A420, 173 (1984).

[20] W. Nazarewicz, Nucl. Phys. A574, 27c (1994).

[21] V.M. Strutinsky, Nucl. Phys. A218, 169 (1974).

[22] P.E. Nemirovsky and Yu.V. Adamchuk, Nucl. Phys. 39, 551 (1962).

[23] W. Satuła et al., to be published.

[24] S. Shlomo, Nucl. Phys. A539, 17 (1992).

[25] S. Frauendorf and V.V. Pashkevich, Ann. Phys. 5, 34 (1996).

[26] A similar conclusion can be drawn based on the seniority model.

[27] N. Zeldes et al., Mat. Fys. Skr. Dan. Vid. Selsk. 3, No. 5 (1967).

[28] A.S. Jensen et al., Nucl. Phys. A431, 393 (1984).

[29] W. Satuła et al., Phys. Lett. 407B, 103 (1997). 